\title{
Finite Element Stimulation Analysis on Osteogenic Capacity of the Gelatin-Hybridized Calcium Phosphate Cement
}

\author{
Tadaaki Morotomi*, Tomomi Iuchi, Tomokazu Fukuda, Kohei Ichinohashi, Hitoshi Nishiwaki, Koji Niwa \\ and Noritaka Isogai
}

Department of Plastic and Reconstructive Surgery, Kindai University Faculty of Medicine, Japan

Received: March 01, 2018; Published: March 14, 2018

*Corresponding author: Noritaka Isogai, Department of Plastic and Reconstructive Surgery, Kindai University Faculty of Medicine, Osaka-sayama, Japan, Email: isogai@med.kindai.ac.jp

\section{Introduction}

When a bone defect is caused due to craniotomy, skull reconstruction is performed with titanium plates or hydroxyapatite in order to protect the brain and achieve an esthetically favorable outcome [1].These non-biodegradable osteo-inducing materials remain in the body and are never incorporated into the auto logos bone. Skull reconstruction using these materials often evokes postoperative complication such as protrusion, osteomyelitis, and instability. The use of biodegradable osteo-inducing biomaterial that can be spontaneously changed to the antilogous bone would be ideal for skull reconstruction; however, such biomaterial has not been developed yet. We previously developed an inject able, biodegradable osteo-inducing biomaterial (gelatin-hybridized calcium phosphate cement, gelatin-hybridized CPC, Gunze Ltd., Kyoto, Japan), and demonstrated its estrogenic capacity using a rat model with a full-thickness skull defect. In the present study, we performed a histological analysis to investigate the influences of the degree of cross-linking and volume of gelatin contained within gelatin - hybridized CPC on the osteogenic capacity. Furthermore, we performed finite element simulation analysis to examine the bone strength of the regenerated bone.

\section{Materials and Methods}

The study was conducted in accordance with the ethical requirements of the Animal Care and Use Committee at the Faculty of Medicine, Kindai University. Ten-week old male SD rats $(n=18)$ were used in the experiments. Rats were raised in clean racks at $22^{\circ}$ and $50 \%$ humidity under a 12 -hour dark-light cycle. They were fed irradiated ( $3 \mathrm{mGy}$ ) pellets, and had unlimited access to drinking water. Following anesthesia with is of lurane, two cylindrical defects ( $5 \mathrm{~mm}$ in diameter) were made on each side of the cranium using a drill. Then, gelatin-hybridized CPC was injected to fulfill the bone defect which became hardened within 5 minutes following injection. Spherical gelatin particles $(50 \sim 100 \mu \mathrm{m}$ in diameter) contained in the gelatin-hybridized CPC were prepared by crosslinking for either 3 or 14 hours (low and high degree of cross- linking, respectively). Furthermore, the gelatin-hybridized CPC was prepared with either 10 or $20 \mathrm{wt} \%$ gelatin. Thus, 4 different groups of gelatin-hybridized CPC were prepared according to the degree of gelatin cross-linking and volume of gelatin contained in the bio degradable osteo-inducing material (Figure 1). The initial compressive strength of gelatin-hybridized CPC was measured for each group prior to implantation. For the compressive strength test, the set cement pastes were packed in cylindrical Teflon $\AA$ molds $\left(6 \mathrm{~mm}\right.$ in diameter and $12 \mathrm{~mm}$ in height), maintained at $37^{\circ} \mathrm{C}$ Cat $100 \%$ humidity for $1 \mathrm{hr}$, and kept in distilled water at $37^{\circ} \mathrm{C}$ for $24 \mathrm{hr}$. The compressive strength tests were performed on the cement specimens using a universal testing machine (AG-5kNXplus, Shimadzu Co., Kyoto, Japan) in accordance with JIS T 0330-4. The crosshead speed was $0.5 \mathrm{~mm} / \mathrm{min}$ and a load cell of $5 \mathrm{kN}$ was used. Tissues were extracted 10 weeks after implantation for histological assessment of the estrogenic capacity. In addition, finite element simulation [2]. was performed using $\mu \mathrm{CT}$ to examine the degree of compression fracture after applying mechanical stress on top of the rat skull.

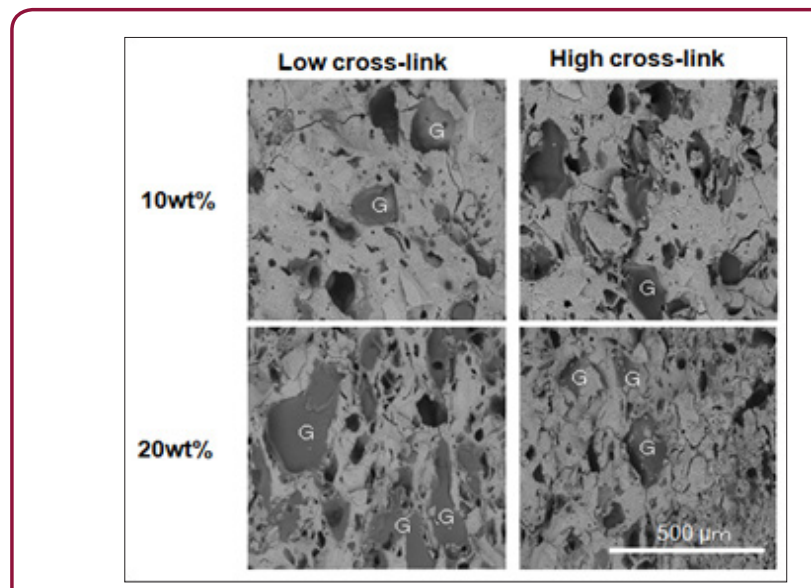

Figure 1: Scanning electron microscope images of the experimental groups. "G" indicates gelatin particles. 


\section{Statistical Analysis}

The Student $t$ test was used to compare 2 means, and the Oneway analysis of variance and the Holm post hoc test were used to compare $>3$ means. All statistical analyses were performed using $\mathrm{R}$ Environment ( $\mathrm{R}$ Project) and $\mathrm{p}<0.05$ was considered to be statistically significant.

\section{Results}

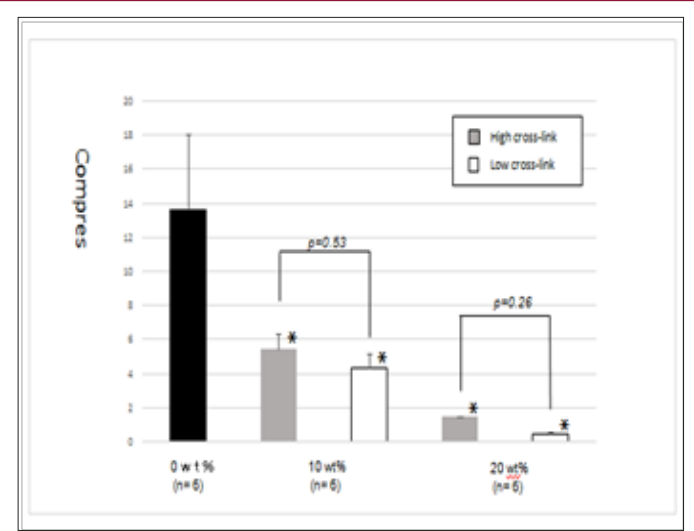

Figure 2: Initial compression strength of the gelatinhybridized CPC prior to implantation. Statistical significance was determined at $\mathrm{p}<0.05\left(^{*}\right)$ level for specimens compared with $0 \mathrm{wt} \%$ and for each group of samples compared between 10 and $20 \mathrm{wt} \%$.

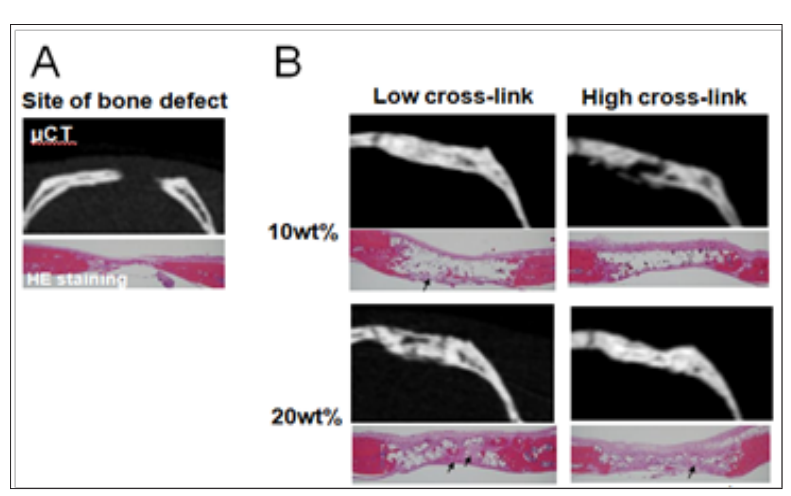

Figure 3:

A. Control: the site of the bone defect 10 weeks after surgery.

B. Histology and $\mu \mathrm{CT}$ images of experimental groups 10 weeks after implantation. The arrows indicate the areas of bone regeneration.

The compressive strength of the hardened, gelatin-hybridized CPC was examined prior to its implantation. The compressive strength of the gelatin-hybridized CPC with 10 and 20wt\% gelatin was significantly lower than that with $0 \mathrm{wt} \%$ gelatin, being approximately $1 / 3$ and $1 / 9$ of the $0 \mathrm{wt} \%$ group, respectively. The degree of cross-linking did not show a significant influence on the compressive strength (Figure 2). By histology and $\mu \mathrm{CT}$, bone regeneration was not completely achieved in the full-thickness skull defect 10 weeks after implantation of the gelatin-hybridized $\mathrm{CPC}$. Bone regeneration was observed more frequently in rats that received the gelatin-hybridized CPC containing $20 \mathrm{wt} \%$ gelatin with a low degree of cross-linking compared with those that received the gelatin-hybridized CPC containing $10 \mathrm{wt} \%$ gelatin with a high degree of cross-linking. Thus, the gelatin-hybridized CPC containing $20 \mathrm{wt} \%$ gelatin with a low degree of cross-linking incorporated more effectively into the antilogous bone (Figure 3). Furthermore, we performed finite element simulation analysis and demonstrated that, following implantation, the bone strength of the rat skull was the greatest in rats that received gelatin-hybridized CPC containing 20wt\% gelatin with a low degree of cross-linking (Figure 4).

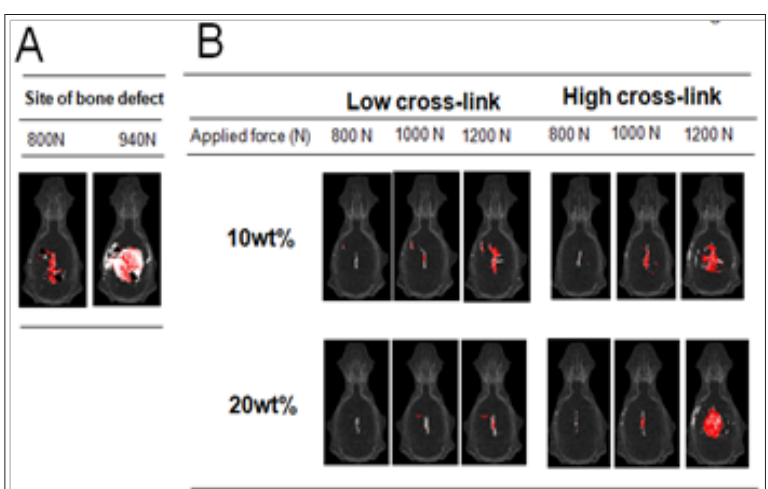

Figure 4: Bone strength of rat skull examined by finite element simulation analysis. Bone strength was measured by finite element simulation analysis after applying a mechanical force to the top of the rat skull. Red indicates the areas of compression fracture, and white indicates the areas of tensile fracture.

A. Bone strength in the control. Bone fracture and depressed deformation were observed in a large area with 940-N mechanical stress.

B. Bone strength in the experimental groups 10 weeks after implantation.

\section{Discussion}

Previous studies examined the osteogenic capacity of gelatincontaining conventional $\mathrm{CPC}$ using rabbit femur and canine mandibular bone defect models. These studies demonstrated a positive correlation between the volume of gelatin contained within CPC and cell infiltration after implantation. However, studies have also shown that the bone strength of CPC is significantly reduced with an increasing volume of gelatin [3,4]. In this study, the strength of CPC was maintained by adjusting the degree of cross-linking and volume of gelatin. Additionally, since gelatinhybridized CPC is inject able, it facilitates casting and can be shaped to fit various forms of bone defects. Our findings suggest that for gelatin-hybridized $\mathrm{CPC}$, the use of a high volume of gelatin with a low degree of cross-linking promotes ontogenesis to improve its incorporation into antilogous bone. It has been reported that about 600 gram of mechanical stress is applied subcutaneously [5]. Thus, the initial strength of the gelatin-hybridized CPC containing 20wt $\%$ gelatin was not sufficient to withstand the stress.

However, finite element simulation analysis demonstrated that the degree of compression fracture was the lowest in defects implanted with gelatin-hybridized CPC containing 20wt\% gelatin 
with a low degree of cross-linking, thus achieving the highest bone strength following implantation. Furthermore, histological analysis of the bone defect revealed that the gelatin particles contained in the gelatin-hybridized CPC were degraded soon after implantation. This suggests that the gelatin-hybridized CPC promotes bone formation by providing a space that facilitates angiogenesis and cell infiltration. Recent studies demonstrated cranium reconstruction using hydroxyapatite produced by a 3D printing technique as well as poly-methyl-methacrylate (PMMA) produced by computeraided design and manufacturing (CAD/CAM) [6]. However, none of the materials are without limitations regarding their use as a biodegradable osteo-inducing biomaterial for skull reconstruction. We demonstrated that the gelatin-hybridized CPC induces ontogenesis at an early stage, enabling the regenerated bone to be incorporated into antilogous bone. Further studies are warranted to examine its use in clinical practice.

\section{References}

1. Zanotti B, Zingaretti N, Verlicchi A (2016) Cranioplasty: Review of Materials. J Craniofac Surg 27: 2061-2072.

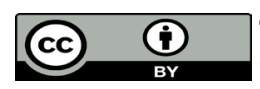

This work is licensed under Creative Commons Attribution 4.0 License

Submission Link: https://biomedres.us/submit-manuscript.php
2. Li K, Wang J, Liu S (2015) Biomechanical behavior of brain injury caused by sticks using finite element model and Hybrid-III testing. Chin J Traumatol 18: 65-73.

3. Kasuya A, Sobajima S, Kinoshita M (2012) In vivo degradation and new bone formation of calcium phosphate cement-gelatin powder composite related to macro-porosity after in situ gelatin degradation. J Orthop Res 28: 1103-1111.

4. Matsumoto G, Sugita Y, Kubo K, Yoshida W, Ikada Y, et al. (2014) Gelatin powders accelerate the resorption of calcium phosphate cement and improve healing in the alveolar ridge. J Biomater Appl 30: 1316-1324.

5. Seno H, Muraishi S, Yanai A (1995) Pressure monitoring of infrared tissue expanders. Jap J Plast Surg 38: 621-628.

6. Unterhofer C, Wipplinger C, Verius M (2017) Reconstruction of large cranial defects with poly-methyl-methacrylate (PMMA) using a rapid prototyping model and a new technique for intraoperative implant modeling. Neurol Neurochir Pol 51: 214-220.

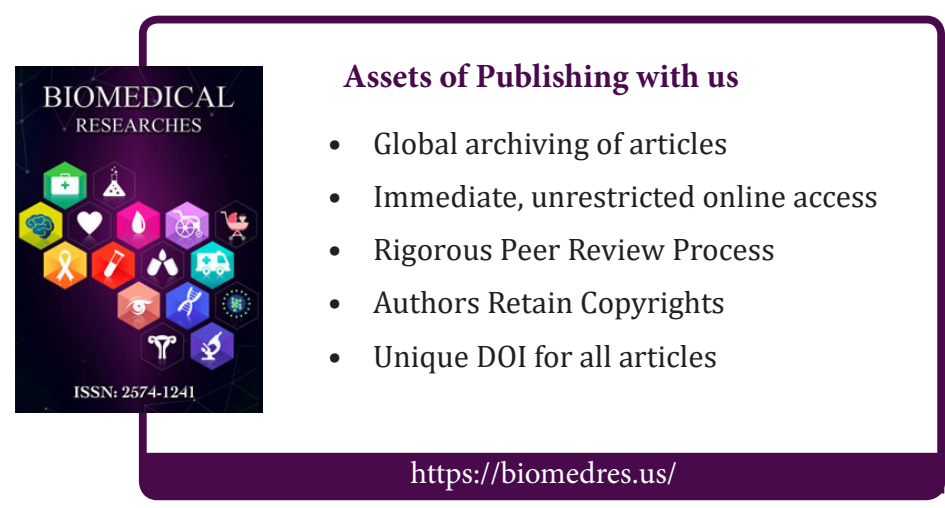

